\title{
The Public Health Challenge of Ending Malnutrition: The Relevance of the World Health Organization's GINA Database
}

Asia Pacific Journal of Public Health

(C) 2018 APJPH

Article reuse guidelines: sagepub.com/journals-permissions DOI: 10.1 I77/1010539518800341 journals.sagepub.com/home/aph

@SAGE

\author{
Fanny Buckinx, MSc, PhD'
}

\begin{abstract}
In order to address malnutrition, implementation of nutrition actions is needed. While academic institutions will certainly play a part, strong coordination and leadership from major United Nations agencies are needed. To this effect, the World Health Organization has launched a Global Database on the Implementation of Nutrition Action (GINA). It contains information collected from various sources, including direct submissions by users. Users can share information on how programs are implemented, including country adaptations and lessons learnt. GINA allows users to share implementation experience in order to identify overlaps and gaps, to foster discussion toward improved planning and to promote good practices. The aim of this article is to highlight the relevance of the GINA database in meeting the public health challenge of ending malnutrition and, therefore, to encourage users from different countries to enter programs and policies in the database.
\end{abstract}

\section{Keywords}

database, GINA, malnutrition, nutrition policies, WHO

\section{Introduction}

Malnutrition management remains a challenge despite worldwide attempts to combat it via policies and programs, which did not reach the expected impact, especially in some of the less developed countries. Likely challenges on scaling up nutrition management are (1) enabling environment, which includes knowledge and evidence; politics and governance; leadership, capacity, and financial resources; social, economic, political, and environmental context; (2) keeping pace with scale-up ambitions; and (3) the funding gap versus urgent resource mobilization and allocation. Several factors could be contributory toward this, and the GINA (Global Database on the Implementation of Nutrition Action) has been conceived with the idea of consolidating country experiences and sharing success stories on a global platform so that best practices and benefits could be available for concerned countries to adopt. Experience with national nutrition policies in the World Health Organization (WHO) European Region has shown the

\footnotetext{
'Department of Public Health, Epidemiology and Health Economics, University of Liège, Liège, Belgium
}

\section{Corresponding Author:}

Fanny Buckinx, Department of Public Health, Epidemiology and Health Economics, WHO Collaborating Centre for Public Health Aspects of Musculoskeletal Health and Ageing, University of Liège, CHU-Sart-Tilman, B23, Quartier Hôpital, Avenue Hippocrate, I3, Liège 4000, Belgium.

Email: fanny.buckinx@uliege.be 
intrinsic value of having shared or common tools and a focus on knowledge translation and transfer. The aim of this article is to highlight the relevance of the GINA database in meeting the public health challenge of ending malnutrition and, therefore, to encourage country users to enter programs and policies in the database.

\section{Methodology}

\section{The GINA Database}

The WHO has launched the GINA database. As indicated on the website,

The purposes of GINA are to collect key data on nutrition policy and action in a standard format, to visualize what is happening where, when, with whom and how, to compare policy commitments with implemented action, to map action and policy against nutrition indicators, to relate action data to the e-Library of Evidence for Nutrition Actions, to link with other databases and mapping tools, and to promote good practice. It can help planners overcome implementation obstacles by learning from other countries' best practices. GINA furthermore provides a repository of country policy commitments and implemented actions. ${ }^{1}$

\section{Description of GINA Database}

The WHO provides an interactive platform for sharing standardized information on nutrition policies and action (ie, what are the commitments made and who is doing what, where, when, why, and how [including lessons learnt]). Users can apply this tool to map nutrition policies and action, link policies and action to nutrition status indicators, monitor implementation of key nutrition action, identify overlaps and gaps, or share experience on implementation practices (http://www.who.int/nutrition/gina/en/).

As you can read on the website,

The GINA policy section tracks commitments to ensuring good nutrition demonstrated in policy and legislative documents. The documents are sometimes specific to nutrition; e.g., national nutrition policies, vitamin A strategies, or codes of marketing of breast-milk substitutes. Other times they have a broader scope; e.g., health or agriculture sector strategies, or development plans. They include policies, strategies, action plans and legislation. The GINA action section tracks action taken to improve nutrition as implemented through programmes and interventions. The programmes are userdefined and can contain an unlimited number of predefined nutrition actions, including both nutritionspecific and nutrition-sensitive interventions. Pre-defined actions range, for instance, from feeding of low-birth-weight infants to implementation of maternity protection, and cover all age groups.

GINA contains information collected from a variety of sources and invites users to directly submit their data. GINA action data can be uploaded by those involved in nutrition interventions, for example, program planners, government officials, nongovernmental organization staff, research teams, or other stakeholders, through a wiki-type approach. Users can share information on how programs are implemented, including country adaptations and lessons learnt. The wiki approach means that contributors can either enter new data or propose edits to existing data, with each submission triggering a verification process. $^{2}$

\section{Data Collected}

On October 15, 2016, the database included 1567 policies in 187 countries.

For each policy, information on national commitments related to the Global Nutrition Targets ${ }^{1}$ 2025 and the Global Monitoring Framework was extracted to a spreadsheet: start date, end date, 
Table I. Number of Actions Implemented in Each Country That Has Submitted Data.

\begin{tabular}{lr}
\hline Action Implemented & $\mathrm{n}$ \\
\hline School feeding programs & 163 \\
Dietary goals and food-based dietary guidelines & 144 \\
Vitamin A supplementation & 125 \\
Food distribution/supplementation for prevention of acute malnutrition & 114 \\
Breastfeeding promotion and/or counselling & 112 \\
Complementary feeding promotion and/or counselling & 101 \\
Iron supplementation & 101 \\
Implementation of legislation on marketing of unhealthy foods and beverages to children & 99 \\
Iron and folic acid supplementation & 91 \\
Growth monitoring and promotion & 85 \\
\hline
\end{tabular}

year of publication, publisher, the type of policy, the country, the partners in policy implementation, the goals, the strategies, the indicators, and the policy topics. The URL link and the PDF document were added.

Indicators related to Global Nutrition Targets were stunting (0-5 years), anemia, low birth weight, child overweight (0-5 years), exclusive breastfeeding (for 6 months), and wasting (0-5 years). In parallel, indicators related to Global Monitoring Framework for Nutrition were underweight in women (body mass index $<18.5$ ); complementary feeding: minimum acceptable diet, overweight and obesity in adults, overweight and obesity in school-age children and adolescents; iron folic acid supplementation to pregnant women; breastfeeding counselling; Baby-Friendly Hospital Initiative; nutrition professionals; International Code of Marketing of Breast Milk Substitutes; and maternity protection. Finally, additional diet-related Global Noncommunicable Disease Targets and indicators of the monitoring framework were encoded: raised blood pressure, raised blood glucose/diabetes, raised total cholesterol, saturated fat intake, salt/sodium intake, and fruit and vegetable intake.

The top 10 actions implemented are listed in Table 1. As can be seen, the actions are varied; a total of 163 school feeding programs have been implemented around the world.

Ghana, India, and Bangladesh are the 3 countries with the most actions published, whereas Costa Rica, Honduras, and Guatemala are the 3 countries with most policies published (Table 2).

\section{Ethics}

The Work Programme of the Nutrition Decade calls on Member States, regional political and economic communities, and the global community to translate the commitments made through the International Conference on Nutrition 2 Rome Declaration on Nutrition into SMART (specific, measurable, assignable, realistic, time-related) commitments for action, in the context of national nutrition and nutrition-related policies. The commitments are being made available in this publicly accessible repository managed by the joint Food and Agriculture Organization/ WHO Secretariat. A single form is completed online by the users, and the encoded data are verified by a member of the WHO staff before being published in GINA.

\section{Results}

\section{How to Enter Programs and Policies in GINA Database?}

To submit data, the first step is to create a GINA account. To do this, WHO staff can use their usual username and password while non-WHO staff have to create an account. Non-WHO staff 
Table 2. The 10 Countries With the Most Actions and Policies Published.

\begin{tabular}{lc}
\hline Countries & Number of Actions Published \\
\hline Ghana & 59 \\
India & 50 \\
Bangladesh & 49 \\
Mexico & 46 \\
China & 45 \\
Thailand & 45 \\
Sri Lanka & 44 \\
The Philippines & 44 \\
Ethiopia & 44 \\
Tavulu & 42 \\
\hline Countries & Number of Policies Published \\
\hline Costa Rica & 52 \\
Guatemala & 41 \\
Honduras & 40 \\
El Salvador & 38 \\
United Republic of Tanzania & 37 \\
Nicaragua & 36 \\
Brazil & 34 \\
Zambia & 30 \\
Bolivia & 30 \\
Peru & 29 \\
\hline
\end{tabular}

need to provide name and affiliation and then to accept the terms and conditions of use for the GINA platform. Then, an administrator must approve the demand to enable contributors to submit new data, to edit/update any published information. As long as the information is not submitted it will remain a draft and is only accessible to the contributor. After submitting the data the regional approver, corresponding to the country to which the data refer, is automatically requested to review the new data.

\section{Limitations}

Website visitors do not have access to what is consulted and how the information of the GINA database is used by other users of the program or policy, which is the main limitation of the GINA database. The number of programs and policies uploaded in the database is only accessible to managers of the database (ie, WHO staff). Moreover, the adoption of policies is unclear. Obtaining this information would be an advantage for the public health challenge of ending malnutrition.

\section{Conclusions}

Continuing or widening of economic disparities results in the persistence of pockets of undernutrition in regions or neighborhoods characterized by poverty and poor access to resources. Increasingly, obesity is affecting low-income populations in urban centers, contributing to the challenges faced by national health care systems.

The GINA developed by the WHO is a tool that could help dealing with all forms of malnutrition, which is a public health priority. 


\section{Acknowledgments}

The author thanks the World Health Organization for the welcome it provided during a 6-month internship in the department of Nutrition for Development and Health.

\section{Declaration of Conflicting Interests}

The author declared no potential conflicts of interest with respect to the research, authorship, and/or publication of this article.

\section{Funding}

The author disclosed receipt of the following financial support for the research, authorship, and/or publication of this article: The author thanks the "Fonds National pour la Recherche Scientifique" for supporting the internship at the World Health Organization.

\section{References}

1. World Health Organization. Nutrition: global targets 2025. http://www.who.int/nutrition/global-target-2025/en/. Accessed August 27, 2018.

2. World Health Organization. Nutrition: global database on the implementation on nutrition action (GINA). http://www.who.int/nutrition/gina/en/. Accessed August 27, 2018. 